\title{
Status of the ALICE Magnet System
}

\author{
D. Swoboda, L. Leistam, L. Pigni, D. Cacaut, E. Kochournikov, and A. Vodopianov
}

\begin{abstract}
Alice is the LHC experiment dedicated to heavy ion $(\mathrm{Pb}, \mathrm{Ca})$ physics. Alice will be installed in point 2 of $\mathrm{LHC}$, the former point 2 of LEP. The luminosity at the interaction point will be in the order of $10^{27}\left(10^{31}\right.$ for $\left.p-p\right)$. Consequently, the radiation level in Alice will be comparatively low. We will use the existing infrastructure. Therefore, no noticeable civil engineering work will be necessary. Minor modifications will be made to gain a second access to the experiment cavern-mainly for the muon arm spectrometer. ALICE will reuse the $L 3$ solenoid, which is already installed and has been used in LEP as spectrometer magnet. A dipole magnet with a very large aperture is in addition required for the Dimuon Arm spectrometer and will be installed next to the $L 3$ solenoid. The detectors in Alice are concentrated around the IP inside L3 (Barrel) and along the muon arm. Some small forward detectors will be located toward the injection area at a larger distance from IP $(\sim 100 \mathrm{~m})$. After a review of the present status of both projects some important milestones will be highlighted in the conclusion.
\end{abstract}

\section{INTRODUCTION}

A LICE is the dedicated heavy ion physics ( $\mathrm{Pb}, \mathrm{Ca}$ ) Experiment for LHC. The installation in Point 2 of the LHC accelerator will be integrated in the existing infrastructure.

The luminosity will range from $10^{27}$ for $\mathrm{p}-\mathrm{p}$ collisions to $10^{31}$ for heavy ions. ALICE will be equipped with a range of different detectors. The detectors are for the majority located inside the L3 Solenoid (Barrel). The large single arm DiMuon forward detector occupies the experimental hall space to the downstream side of L3. Some smaller forward detectors are located upstream at distances up to about $100 \mathrm{~m}$ from IP.

The preparation of the experimental site also requires the dismantling of the L3 experiment. This task is in progress and will be accomplished during the year 2001. The subsequent installation of the ALICE experiment will include a revision of the L3 solenoid, the magnet control system and the electricity supply.

The L3 solenoid has been in operation since 1988 throughout the LEP era. The previously installed detectors are now dismantled and the magnet will undergo substantial preventive maintenance work and modifications to conform to the requirements for the ALICE detectors. The extent of the corrosion detected in the coil cooling circuits is difficult to evaluate. Therefore, a partial reinforcement of the piping will be undertaken. The principal problems of this paper will be described together with the proposed solutions.

Some central detectors and specifically the TPC require homogeneity of the magnetic field better than the present values. Modifications to the magnetic circuit of the solenoid magnet are therefore necessary to obtain a good field quality.

Manuscript received September 24, 2001.

D. Swoboda, L. Leistam, and L. Pigni are with CERN, Geneva, CH.

D. Cacaut is with INFN, Alessandria, IT.

E. Kochournikov and A. Vodopianov are with JINR, Dubna, RU.

Publisher Item Identifier S 1051-8223(02)03579-0.
A second large dipole magnet is required for the muon arm spectrometer. This magnet will be placed directly adjacent to the L3 solenoid magnet.

The Muon Arm spectrometer requires a large Dipole Magnet for particle identification. In March 1997 the decision was taken to build a resistive dipole magnet. The absence of criteria for the necessary symmetry and homogeneity of the magnetic field has lead to a design dominated by economical and feasibility considerations.

The general concept of the dipole magnet is based on a window frame return yoke, fabricated from low carbon steel sheets. The flat vertical poles follow the defined acceptance angle of 9 degrees. The excitation coils are of saddle type.

The coils are wound from large hollow Aluminum profiles. They are cooled by pressurized demineralized water. The coil ends are located on both sides of the magnet yoke and determine the overall length of the magnet. The main flux direction in the gap is horizontal and perpendicular to the LHC beam axis.

The design work has been pursued in JINR/Russia and at CERN. The production of the excitation coils and the return yoke of the magnet has started this year. However, some ancillary systems are still under development or in the process to be specified.

The employed manufacturing technology for the main items will be described and the incidence on the design choices will be explained.

The design has now been terminated and the manufacturing has started. The magnet will be powered by a dc power supply. The coil will be water-cooled with demineralized water.

\section{L3 SOLENOID MAGNET}

The magnet is described in the ALICE TP [1]. It has been operated throughout the time of LEP physics Fig. 1. An upgrading of the electrical and cooling supply is now necessary to prepare the coming running period of at least 10 more years.

The modifications of the power converter will principally concern new control electronics to be compatible with the LHC electricity system. This is a routine intervention, which is scheduled during the installation period of the experiment.

The modifications in the main cooling system are meant to improve the cooling capacity in order to compensate in the event of partial failure of the magnet cooling system. Presently, the temperature of the cooling water inlet varies with the outside conditions and can reach up to about $25{ }^{\circ} \mathrm{C}$. An additional heat exchanger will therefore be installed in order to maintain a stable inlet temperature of $20{ }^{\circ} \mathrm{C}$.

A project for the supply of a common control system for all LHC experiment magnets has been launched. It is therefore also planned to replace the L3 magnet control system by that common system. 


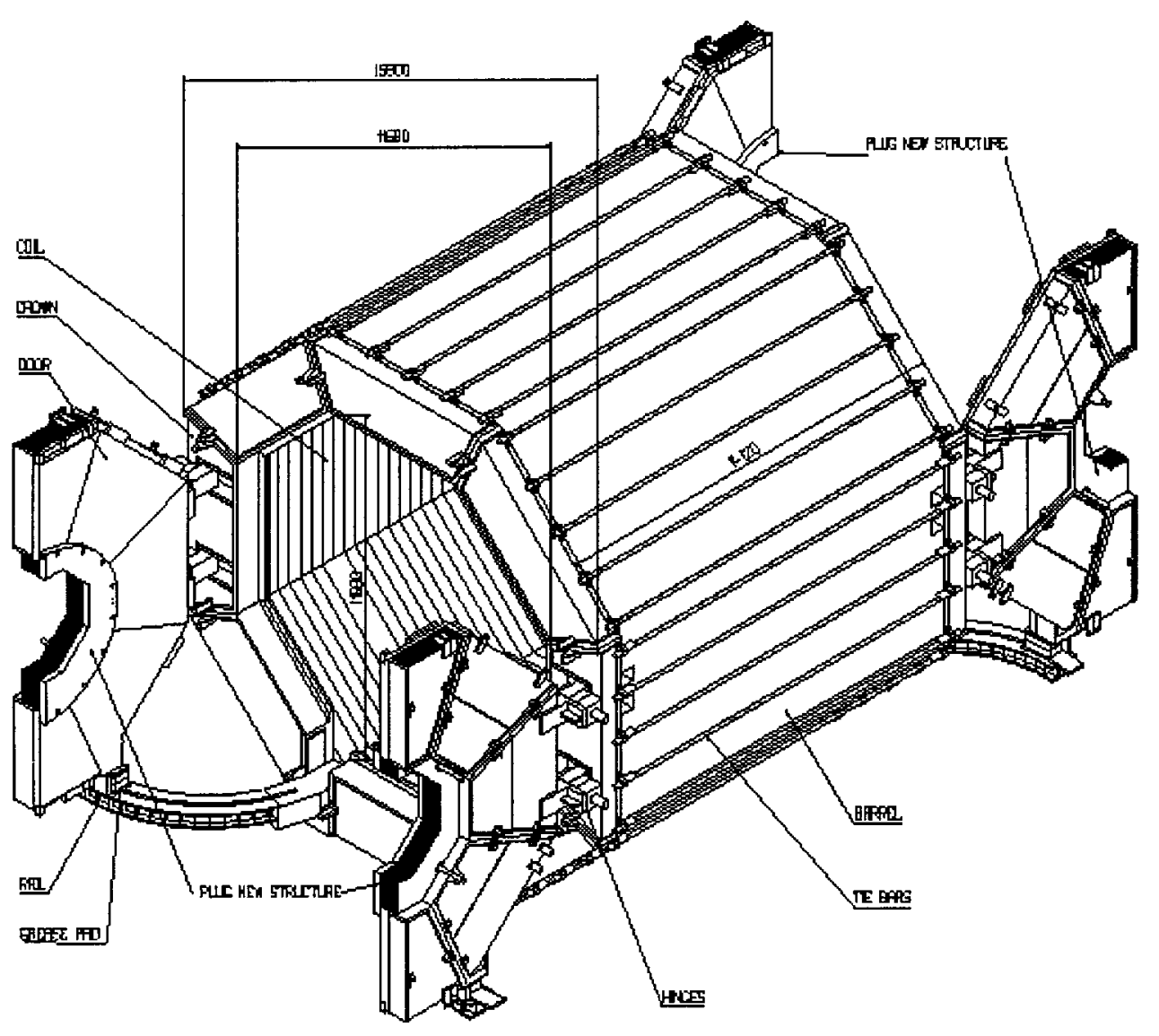

Fig. 1. L3 solenoid magnet.

\section{A. Cooling Circuit Maintenance}

The coil of the L3 magnet consists of 168 octagonal turns constructed from 60-mm-wide and 890-mm-thick aluminum plates. The plates are assembled into turns by electron beam welding and grouped in 28 packages of 6 turns each. All turns are electrically connected in series. The electrical connections are made at the upper horizontal plates of the turns. Each of these connection bars has a separate cooling circuit.

Each turn has an inner and an outer cooling circuit. These circuits consist of hexagonal aluminum tubes, which are welded directly to the inner and outer perimeter of each turn and joint at each corner of the octagon by welded cylindrical aluminum tubes. One hydraulic circuit extends over 3 turns, adding up to a total of 56 inner and 56 outer circuits.

After the discovery of considerable corrosion at one cooling circuit in 1991 a number of studies and tests have been performed to analyze the origin and extent of the degradation. It was concluded that the weak regions are the joints between hexagonal and cylindrical cooling tubes. These locations can be accessed at the inner perimeter of the coil after complete removal of the L3 experiment detectors and dismantling of the thermal shields, which separate the coil from the inner volume of the magnet.

However, the mechanical structure of the magnet precludes any intervention at the outer cooling circuits, which are trapped between flux return yoke and coil.

A project for preventive maintenance has been set up to secure the part of the cooling system which can be accessed. A failure of the outer circuits will therefore still have some effects on the magnet operation. (See Section II-C.)

Several technologies were investigated to reinforce the joint regions. The method, which was retained, consists in an encapsulation of the joints with epoxy resin Fig. 2. This technique has been developed and tested by CERN. Epoxy resin will be injected into lost molds, which will be fitted around the joint regions. This procedure needs to be applied at 2742 connections. In addition it is necessary to replace the pipe work between the cooling water manifolds and the 112 cooling circuits. This requires the fitting of 1000 cylindrical aluminum sleeves and their subsequent reinforcement with epoxy resin.

\section{B. Pole Cap Modification}

The L3 magnet yoke is built as an octagonal steel barrel around the aluminum coil. In order to limit the stray flux at the magnet ends, pole caps of approximately $100-\mathrm{cm}$ thickness are fitted at either side. They are in the form of two sectors per side, which build an octagon with an inner octagonal passage in the center. Each sector is constructed as a hinged "door" which can be pivoted on a grease pad system to provide access to the magnetic volume. The required field homogeneity of some central detectors, especially the TPC in ALICE made it necessary to reduce the opening in the end cap center [2]. Steel-inserts (plugs) will therefore be mounted in the doors Fig. 3. These plugs are designed in a similar construction to the L3 doors. Steel plates are stacked and consolidated by tie rods. 


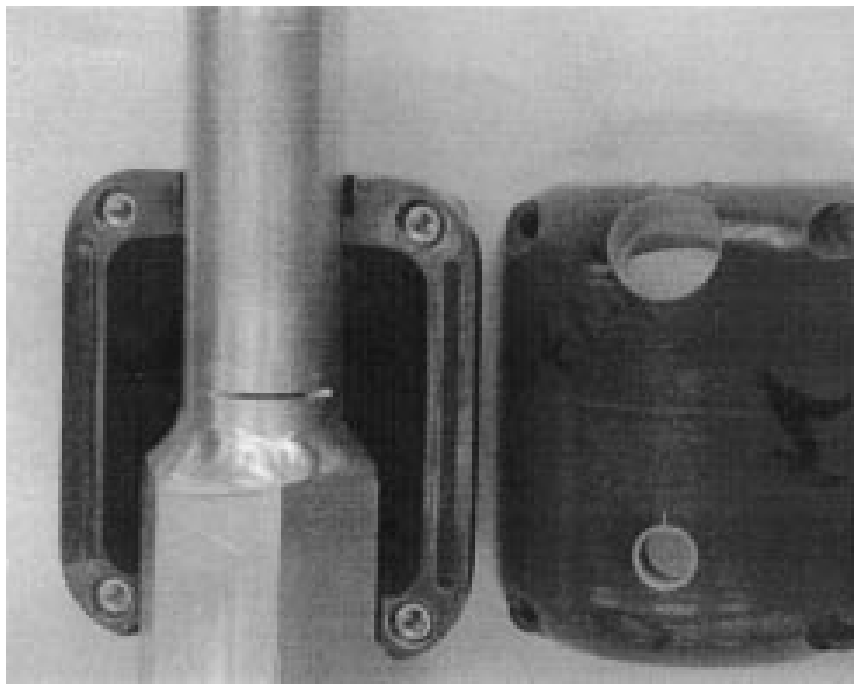

Fig. 2. L3 cooling pipe reinforcement.

The sectors are then fitted inside the hole of the L3 doors and flanged around the inside perimeter. Each insert will add about 40 tons weight to the present weight of approximately 340 tons per door. Since the geometrical length axis of the L3 magnet lies $30 \mathrm{~cm}$ below the beam line for LHC the inserted plugs will have an asymmetric shape to provide an opening with the center at the LHC axis.

\section{L3 Operation}

The scenario of a failure of a hydraulic circuit has been studied. After the final shutdown of LEP, several operating modes were tested in order to evaluate running of the magnet with reduced cooling power.

Since the cooling circuits at the inner perimeter of the coil will be revised, water leaks should only occur in the hydraulic circuits at the outer perimeter of the coil. The tests were done with the magnet powered at reduced current and the outer circuits closed as well as without cooling of the interturn connections Table I.

From the measurements and ANSYS computations it can be concluded that the requested minimum field level of $0.3 \mathrm{~T}$ can be maintained during all conditions. A magnetic field of up to 0.4 $\mathrm{T}$ appears reachable with the improved cooling system in case of partial failure of the hydraulic cooling circuits. The limiting temperature elevation occurs only at the interturn connections, which are located on the top of the coil.

\section{MuON ARM Dipole MAGNET}

The evolution of the conceptual design for the dipole magnet has already been described [3], [4].

The concept of the magnet is based on a window frame return yoke that is fabricated from low carbon steel sheets.

The general layout of the magnet is shown in Fig. 4. The parameters for the approved design are given in Table II.

A complete manufacturing design [5] has been reviewed in July 2000.

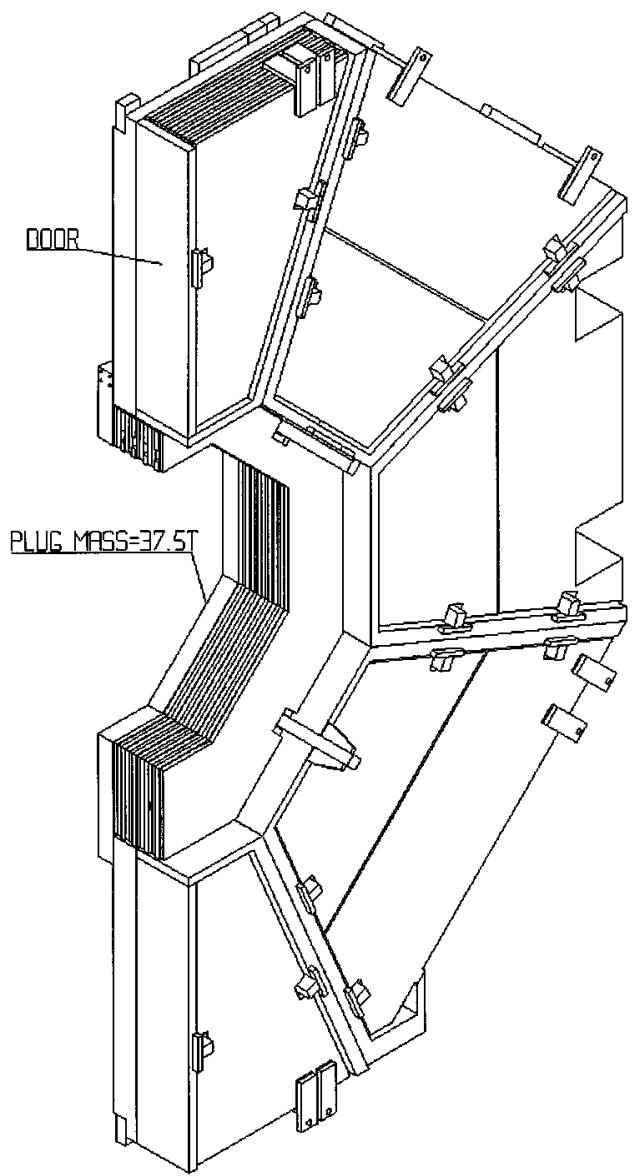

Fig. 3. L3 door plugs.

TABLE I

L3 COOLING Circuit TEMPERATURE

\begin{tabular}{|c|c|c|c|c|}
\hline Current & $\begin{array}{l}\text { Mag } \\
\text { Field }\end{array}$ & $\begin{array}{c}\text { Flow } \\
\text { Inner } \\
\text { Circuit }\end{array}$ & $\begin{array}{c}\text { Flow } \\
\text { Outer } \\
\text { Circuit }\end{array}$ & $\begin{array}{c}\text { Inter } \\
\text { turn } \\
\text { Connect. }\end{array}$ \\
\hline$[\mathrm{kA}]$ & {$[\mathrm{T}]$} & {$[1 / \mathrm{min}]$} & {$[1 / \mathrm{min}]$} & {$\left[{ }^{\circ} \mathrm{C}\right]$} \\
\hline 17.5 & 0.3 & 30 & 0 & 52 \\
\hline 22.5 & 0.4 & 25 & 0 & $>65$ \\
\hline 30 & 0.5 & 25 & 25 & 57 \\
\hline
\end{tabular}

A public tender procedure for the supply of the excitation coils was launched at the end of 2000. The companies to produce the items were selected early 2001. A redesign of the iron yoke and the support structures for coils and magnet was started simultaneously in collaboration between CERN and JINR. This has resulted in an approved design for the iron yoke and the selection of the manufacturer.

In addition, the installation scenario has been modified, which allows some simplifications of the support structure for the magnet but required a revision of magnet dimensions in order to maintain the specified acceptance. The magnet will now be installed on a reinforced concrete foundation, which is in common with several other heavy structures to improve the overall mechanical stability of the system.

The flat vertical poles follow the required acceptance angle of 9 degrees. The excitation coils are of saddle type. They are 


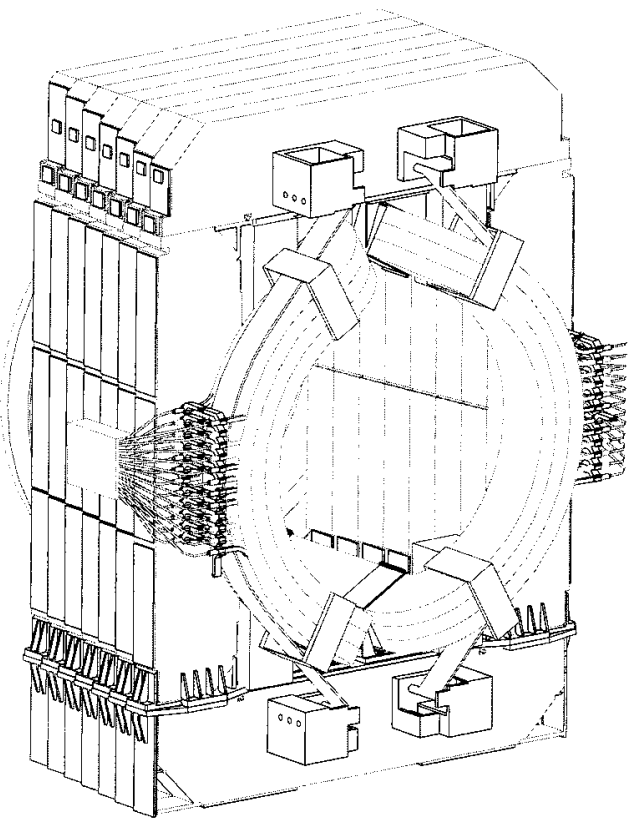

Fig. 4. Dipole magnet assembly.

wound from hollow aluminum conductor of large cross-section. The coil ends are located to both sides of the magnet yoke and determine the length of the magnet.

The main flux direction in the gap is horizontal and perpendicular to the magnet length axis.

\section{A. Magnet Yoke}

In absence of constraints on the field quality the design is focused on low material and manufacturing cost.

The window frame yoke has horizontal bottom and top beams of trapezoidal shape. The poles are formed by vertical sidewalls with the inner surface machined at an angle of 9 degrees to provide the required physics acceptance.

The proposed construction is based on existing steel stacks, which are available from a former cyclotron project at JINR that has been abandoned. The iron yoke is constructed with modules of about $45-\mathrm{cm}$ thickness. Each module consists of $3 \mathrm{~cm}$ thick steel laminations joined under pressure and welded to each other through previously machined holes Fig. 5 .

The modules need to be cut to the required dimensions. The top and bottom parts will be assembled from seven modules each. Each pole will also be made from seven modules. Since the poles are orientated in an angle of 9 degree with respect to the length axis of the magnet it is necessary to machine the sidewalls correspondingly.

The actual dimensions of each of the 28 steel stacks have been cataloged. The difference in thickness exceeds the specified tolerance for the yoke length. The stacks are therefore arranged in order to obtain the same total dimension for each of the yoke subassemblies.

The required inner yoke height of $6 \mathrm{~m}$ exceeds the shorter length dimension of the steel modules. Triangular laminated steel inserts, which are welded at top and bottom of each vertical module will be used to fill the gaps formed at the pole sides. The inserts are designed to serve also as flanges for the connection
TABLE II

MAIN CHARACTERISTICS OF THE MAGNET

\begin{tabular}{|l|l|l|}
\hline Parameter & Value & Unit \\
\hline Max Flux density & 0.67 & $\mathrm{~T}$ \\
\hline Bending Strength & 3.00 & $\mathrm{Tm}$ \\
\hline Avg. Gap width & 3.30 & $\mathrm{~m}$ \\
\hline Ampere turns & 1.97 & $\mathrm{MA}$ \\
\hline Operating Current & 5.86 & $\mathrm{kA}$ \\
\hline Coil Voltage & 590 & $\mathrm{~V}$ \\
\hline Power & 3.46 & $\mathrm{MW}$ \\
\hline Inductance & 1.00 & $\mathrm{H}$ \\
\hline Diff. Pressure & 10.9 & $\mathrm{Bar}$ \\
\hline Diff. Temperature & 30 & ${ }^{\circ} \mathrm{C}$ \\
\hline Total weight & 835 & $\mathrm{Tons}$ \\
\hline $\begin{array}{l}\text { Overall } \\
\text { Dimensions } \\
\text { (H x W x L) }\end{array}$ & $9.0 \times 6.7 \mathrm{x}$ & $\mathrm{m} \mathrm{x} \mathrm{m} \mathrm{x}$ \\
$\mathrm{m}$
\end{tabular}

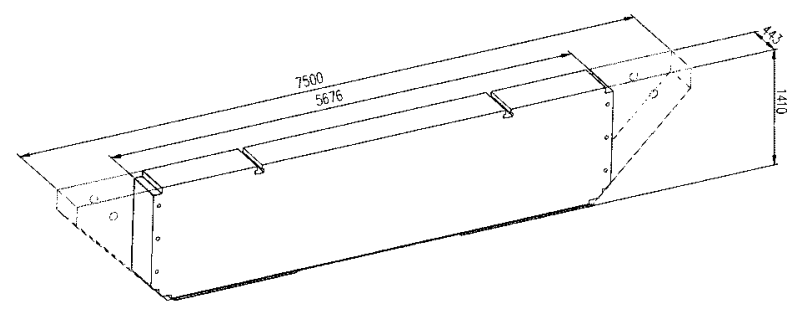

Fig. 5. Actual steel stack dimensions.

of horizontal and vertical modules. They need to be welded to the modules before machining of the mating faces.

The longitudinal connection between modules will be made by means of tie rods. Most of the existing holes in the horizontal modules can readily be used for this purpose. Since the vertical modules are placed in a staggered arrangement to follow the 9 degree angle, new holes need to be machined and the existing holes will be filled with steel plugs.

The proximity of the L3 solenoid to the dipole magnet results in considerable electromagnetic forces in the order of 200 tons between the two magnets. Extensive mechanical calculations have, therefore, been performed [6], [7] to evaluate the mechanical stability of the structure under load. An improvement of the rigidity of the steel stacks appeared to be necessary and it is proposed to weld reinforcement plates at inside and outside of the vertical modules.

\section{B. Coil System}

The general geometry and parameters of the excitation coils have been described in the engineering document [8]. The excitation windings consist of two mirror symmetrical saddle-shape coils. A coil is constructed from 12 pancakes with 14 turns each. The cross-section of the coil is rectangular. The longitudinal parts of a coil are parallel to each other. The coil ends have a semi-cylindrical shape forming an arc with a total angle of 122 degrees. The bends of the coil lie in one plane and transit smoothly into the cylindrical part of the coil ends. The coils will be wound from hollow conductor extruded from $99.7 \%$ purity aluminum. 
In order to entrust the manufacturing to industry the manufacturing technology needed to be adapted to the know-how of the selected supplier.

Therefore, it became necessary to review the initially proposed technology with prepreg insulation tape and opt for a classical vacuum impregnation. The size of the coil volume requires a rather large amount of resin of more than one ton for each coil. Such quantity is very difficult to manage since the impregnation time during which the epoxy needs to be maintained liquid is several days. Each coil has, therefore, been divided into three subcoils of four pancakes each Fig. 6.

Each subcoil will be constructed as a self-consistent assembly including ground wrap insulation.

The thermal expansion of the coil will be of the order of 4 to $5 \mathrm{~mm}$ during magnet operation. Therefore, the coil will slightly move along the longitudinal supports. The saddle parts of the coil will be clamped to the pole ends with the help of ribs and intermediate rubber sheets at the connection side.

Stainless steel bands around the outer perimeter of the coil ends will be used to confine the coils radially.

The electrical connections will be located on the outer perimeter of the coil ends. The connection between adjacent layers will consist of plain aluminum bars of large cross-section with a laminated flexible part in the center Fig. 7. The bus bars are connected to the pancake terminals by stainless steel flanges.

The water inlets will be connected to the water collectors via manifolds and rubber pipes to provide the electrical insulation.

\section{Excitation Windings.}

A continuous extrusion process is used to produce the Aluminum conductor of large cross-section.

Each coil layer constitutes to a separate cooling circuit. A pancake is first wound flat on a mandrel. The winding procedure is carried out with constant tension to maintain the mechanical properties and homogeneity while bending the sections. The conductor will be aligned and straightened simultaneously by applying some tension when pulled through the winding chain equipment. The small inner bending radius of $250 \mathrm{~mm}$ will require compensating the keystone effect. This can be obtained by machining an inverse profile in the concerned region before bending. The conductor insulation can then be applied before bending the conductor during the winding process. The wound flat pancake is finished by applying the layer insulation wrap.

In a second step the flat pancakes are mounted on a cylindrical fixture where they are shaped individually to the semicylindrical form. In the end parts of the winding, the bending radius of each layer is greater than $2 \mathrm{~m}$ and leads only to a very small deformation $(1.25 \%)$ of the conductor cross-section. Consequently, the previously wrapped conductor insulation should not be degraded when bending one coil layer to the 3-D shape.

During the shaping process of a layer, the cylindrical surface of the fixture will be the reference for the inner cylindrical surfaces of each layer.

The spring back needs to be taken into account during the shaping process. The bending radius must therefore be adapted correspondingly. Some tests have already been performed to evaluate this effect [9].

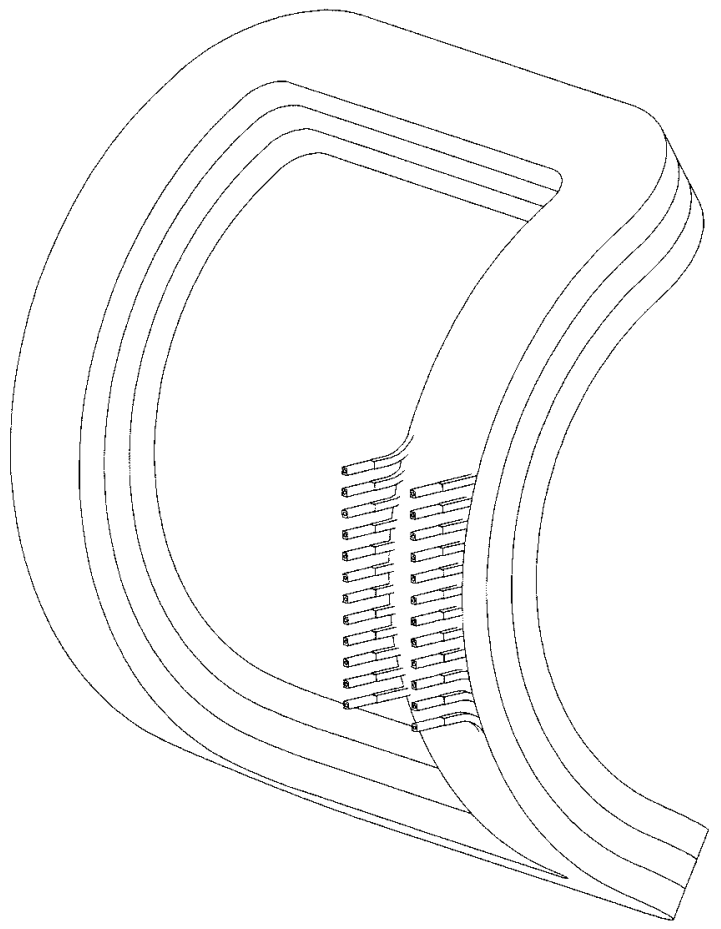

Fig. 6. Coil assembly.

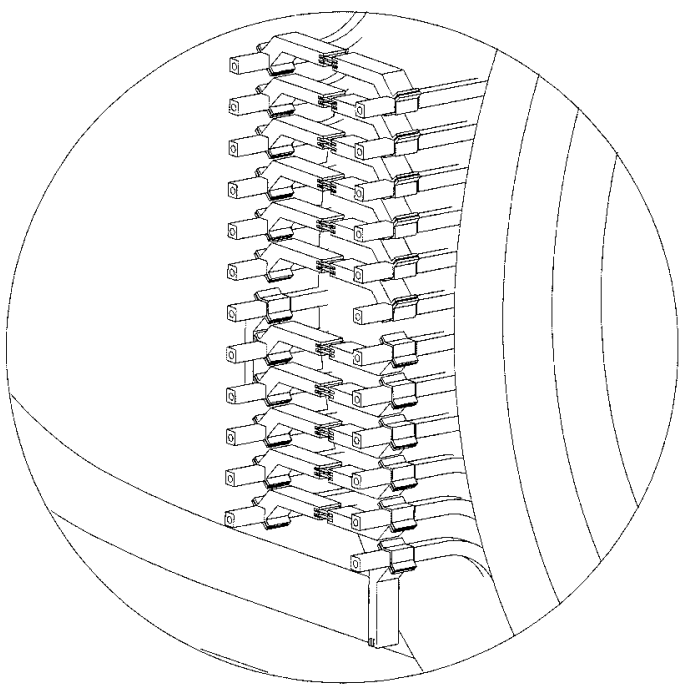

Fig. 7. Electrical connections.

The four pancakes of one subcoil need to be stacked on an adequate support jig to apply the ground wall insulation. Subsequently the assembly must be transferred and inserted into the impregnation mold. This process is crucial since the quality of the impregnated subcoil will depend on the geometrical precision of the stack. The subsequent production steps correspond to a traditional vacuum impregnation. Care must be taken, however, to guarantee a good epoxy penetration of the glass fiber insulation and control vacuum and temperature during the curing cycle.

The final assembly of the subcoils will be done before the insertion into the magnet yoke. Temporary steel bandages are foreseen to confine the coils to the specified dimensions. These bandages will be removed after the adjustment of the coils in- 
side the support structures. The free movement between the subcoils should also help to reduce the thermal and electromagnetic stresses in the conductors.

\section{Power Supply and Cooling System}

The power converter for the electrical supply of the ALICE dipole magnet is identical to the one ordered for another LHC experiment and will be installed inside a surface building. Already existing aluminum cables will be used to connect the magnet over a distance of about $150 \mathrm{~m}$ length. Additional cooling is therefore no longer required.

Direct water-cooling of the coils will remove the major part of the generated heat in the excitation windings. The water inlet temperature will be stabilized to $20^{\circ} \mathrm{C}$.

The general policy requires that generated heat load is removed at the source. The temperature differential between inlet and outlet of the hydraulic circuits will, however, lead to an average temperature elevation of $15{ }^{\circ} \mathrm{C}$ of the coil surface.

A simple way to shield the environment is suggested by using thermal insulation around the coil perimeter. This method is presently under study and would avoid the installation of costly active heat exchanger panels.

\section{E. Magnet Assembly}

Several successive assemblies of the yoke and of the complete magnet will be necessary. A first assembly of the yoke is required at the manufacturers premises to control dimensions and produce all alignment references. Once this has been successfully terminated, subsequent reassemblies shall not require realignment of the subunits. Whilst the requirements on magnetic field homogeneity are not strong, the large dimensions of the magnet make tight machining tolerances essential. The machined faces at the external sides of the horizontal parts, which are used as references for the mating faces between horizontal beams and uprights, will also serve as support areas on the magnet foundation. Consequently, an alignment of the horizontal beams should be sufficient to guarantee the correct position of the magnet.

Following the decision to make a permanent installation of the magnet on a reinforced concrete foundation in vertical position, a removable connection to the foundation is also no longer required. The foundation will, therefore, be equipped with a steel platform to which the bottom of the yoke can be fixed by welding.

Tension rods will be used to consolidate the horizontal beams and the uprights. Adequately positioned dowel pins will guarantee the alignment between subunits. The mechanical assembly of horizontal and vertical yoke parts has to take the geometry of the yoke into account. To guarantee a precise match at the junction between these items, flanges will be welded at the extremities of the subunits and machined simultaneously with the mating surfaces. The mechanical connection will then be achieved with tension bolts between flanges.
The coils will have to be inserted once the yoke base and the pole sides have been mounted and aligned. Consequently, subsequent realignment and adjusting of tie rod tension will not be possible. Only when the coils are fixed in the final position can the top modules be lowered and connected to the sidewalls of the yoke. This requires also temporary supports for the upper coil fixations to the yoke.

\section{CONCLUSIONS}

The revision and modifications for the L3 magnet constitute a challenging project which needs to be terminated before the installation of the ALICE detectors proper. Several interventions, like control system and cooling circuit modification, interfere with the operation of the magnet. A detailed test program will therefore be established to assure a reliable magnet function thereafter. The modification of the doors and the installation of the dipole magnet will alter the magnetic field inside the solenoid such that a complete remapping of the useful volume will be required. In addition ALICE will run at different field levels and polarities. Several measurement programs are therefore necessary.

The proximity of the magnets leads to stronger requirements for the mechanical rigidity of both magnets. The yoke structure of the dipole magnet therefore needs substantial reinforcements.

The infrastructure and available logistics at the ALICE site at point 2 require a strict and reliable scheduling of the installation. The dipole magnet will need to be assembled and commissioned in the experimental hall within a tight time frame before end 2004 after the overhaul of the L3 solenoid has been terminated. To validate the installation procedure and minimize the risk of unforeseen adaptations at that time, a full assembly and precommissioning in a surface hall at point 2 will precede the final assembly in the experimental cavern. This procedure appears essential to verify the conformity of the different components, which are constructed at distant locations.

\section{REFERENCES}

[1] D. Swoboda, "ALICE muon arm dipole magnet conceptual design report,", ALICE/99-06.

[2] V. I. Klyukhin et al., "The ALICE dipole magnet calculation,", ALICE/96-44.

[3] D. Swoboda et al., "Design and status of the dipole spectrometer magnet for the ALICE experiment," IEEE Trans. Appl. Superconduct., vol. 10, Mar. 2000.

[4] "TDR for the dimuon forward spectrometer," CERN/LHCC 99-22, ALICE TDR 5, Aug. 13, 1999.

[5] "Engineering design and manufacturing specification," JINR, Dubna, ALICE.051 00.006.00TZ, July 2000.

[6] A. Makarov, "Strength analysis of the dipole magnet iron yoke," JINR, 2296.01.000R1, July 2001.

[7] A. Wroblewski, "Remarks on the strength analysis of the dipole magnet iron yoke," Cracow Univ. Technol., Sept. 2001.

[8] "Design technical description of the dipole magnet of the ALICE muon spectrometer," JINR, 2296.00.000TO, July 2000.

[9] W. Flegel et al., "Bending test of conductor for ALICE and LHCb dipole magnets,", ALICE/2000-08, Apr. 2000. 